\title{
Metabolic loading of guanosine induces chondrocyte apoptosis via the Fas pathway
}

\author{
Dong-Jo Kim ${ }^{1,2 \#, ~ J u n-H o ~ C h u n g ~}{ }^{2}$, \\ Eun-Kyeong Ryu ${ }^{3}$, Jung-Hyo Rhim ${ }^{2 *}$, \\ Yoon-Sic Ryu', So-Hyun Park', \\ Kyung Tae Kim ${ }^{4}$, Heun-Soo Kang ${ }^{1}$, \\ Hong-Keun Chung ${ }^{2}$ and Sang Chul Park ${ }^{2,4,5}$ \\ ${ }^{1}$ Metabolic Engineering Laboratory Inc. \\ ${ }^{2}$ Department of Biochemistry and Molecular Biology \\ ${ }^{3}$ Xenotransplantation Research Center \\ ${ }^{4}$ Aging and Apoptosis Research Center \\ Seoul National University College of Medicine \\ Seoul 110-799, Korea \\ ${ }^{5}$ Corresponding author: Tel, 82-2-740-8244; \\ Fax, 82-2-744-4534; E-mail, scpark@snu.ac.kr \\ ${ }^{*}$ Center for Cancer Research \\ $\mathrm{NCl}$, National Institutes of Health \\ Bethesda, MD 20892, USA \\ \#Present address: Department of Anatomy and Neurobiology \\ Washington University School of Medicine \\ St. Louis, MO63110, USA
}

Accepted 4 July 2006

Abbreviations: Fas-Fc, Fas-Fc chimeric protein; FasL, Fas ligand

\begin{abstract}
Although the apoptosis of chondrocytes plays an important role in endochondral ossification, its mechanism has not been elucidated. In this study, we show that guanosine induces chondrocyte apoptosis based on the results of acridine orange/ ethidium bromide staining, caspase- 3 activation, and sub-G1 fraction analysis. The potent inhibitory effect of dipyridamole, a nucleoside transporter blocker, indicates that extracellular guanosine must enter the chondrocytes to induce apoptosis. We found that guanosine promotes Fas-Fas ligand interaction which, in turn, leads to chondrocyte apoptosis. These findings indicate a novel mechanism for endochondral ossification via metabolic regulation.
\end{abstract}

Keywords: apoptosis; chondrocytes; ossification; Fas ligand; guanosine

\section{Introduction}

Endochondral ossification is a process by which a mesenchymal cartilagenous template is replaced by mineralized skeletal elements (Wright et al., 1995). This process is driven by the proliferation and differentiation of growth plate chondrocytes that undergo a number of profound physical and biochemical changes as they mature (Zuscik et al., 2002). Initially, growth cartilage cells proliferate, becoming mature chondrocytes that can form an extracellular matrix (ECM) composed of aggrecan and type II collagen (Poole, 1991). The cells then transform into hypertrophic chondrocytes that are capable of producing type $X$ collagen and alkaline phosphatase (ALPase). The matrix incorporates minerals and blood vessels so that hematopoietic osteoclast-precursor cells and perivascular osteoblastprogenitor cells can be recruited. The cartilage is replaced by bone (Poole, 1991) in the final step of the process. Accompanying these changes is an increase in the number of apoptotic cells. A number of studies support the hypothesis that the terminally differentiated chondrocytes of the growth plate undergo programmed cell death (Gibson, 1998). How apoptosis is initiated and regulated remains to be elucidated.

Another notable change occurring in the cartilage is a significant increase in the guanosine level in the hypertrophic zone and in the calcified cartilage (Matsumoto et al., 1988). At present, the role of guanosine in chondrocytes has not been identified. Nonetheless, natural nucleosides are important metabolites and are the main source for nucleic acid synthesis. Nucleosides also have a myriad of physiological functions in various organs and systems, for example, acting as an anti-inflammatory agent and inhibiting lipolysis in fat cells (Griffith and Jarvis, 1996). The elevation of the guanosine level in the growth plate indicates a potentially significant physiological function for guanosine in chondrocytes. We present evidence that guanosine plays an important role in the apoptotic cell death of the growth plate. Our results indicate that elevation of the guanosine level in chondrocytes induces Fas ligand induction and eventually leads to cellular apoptosis. 


\section{Materials and Methods}

\section{Chemicals and reagents}

Pepstatin A, leupeptin, phenylmethylsulfonyl fluoride, and aprotinin were purchased from Roche Molecular Biochemicals (Basel, Switzerland). Human Fas-Fc and a Cpp32/caspase-3 colorimetric protease kit were obtained from R\&D systems (Minneapolis, MN). FBS was a Gibco-BRL product (Grand Island, NY), and MTT, acridine orange, ethidium bromide, and dipyridamole were purchased from Sigma (St. Louis, MO).

\section{Chondrocyte culture}

A primary culture of chondrocytes was prepared from rat costal cartilage according to the procedure previously described by Izumi et al. (1995). Briefly, 1-week-old Sprague-Dawley rat costal cartilage was placed in DMEM containing $4.5 \mathrm{~g} / \mathrm{L}$ glucose. Cartilage slices were digested in $1 \%$ trypsin followed by $0.1 \%$ collagenase. After rinsing with growth medium (DMEM, 10\% FBS, $50 \mu \mathrm{g} / \mathrm{ml}$ penicillin/streptomycin), a single cell suspension was obtained. Analyses with chondrocytes were peformed by culturing the cells in growth medium for various time periods. The effects of guanosine and other reagents were examined, using the culture medium without the reagent as a control system.

\section{Staining cells with acridine orange/ethidium bromide}

A cover slip was placed in each well of a 6-well culture plate (Nunc, Naperville, IL) and coated with $3 \%$ gelatin. Rat chondrocytes were seeded at $1.0 \times$ $10^{5}$ cells per well in $3 \mathrm{ml}$ of $10 \%$ FBS-DMEM by incubating for $24 \mathrm{~h}$, as described by Folkman et al. (1979). Cells were then cultured in the absence or presence of guanosine for $48 \mathrm{~h}$. The culture medium was removed and $100 \mu \mathrm{l}$ per well of a dye mixture $(100 \mu \mathrm{g} / \mathrm{ml}$ acridine orange, $100 \mu \mathrm{g} / \mathrm{ml}$ ethidium bromide in PBS) was added to the cells. Subsequently, the cover slips were mounted onto glass slides and visualized using a Bio-Rad 2100 MP confocal system (Bio-Rad, Hercules, CE).

\section{Measurement of caspase-3 activity}

The caspase- 3 activity was measured using a Cpp32/ caspase-3 colorimetric protease kit following the manufacturer's instruction. Briefly, $1 \times 10^{7}$ cells were lysed on ice for $30 \mathrm{~min}$ with $100 \mu \mathrm{l}$ of chilled 'cell lysis buffer' included in the kit. After centrifugation at $12,000 \times \mathrm{g}$ for $15 \mathrm{~min}$ at $4^{\circ} \mathrm{C}$, the protein concentration in the supernatant was determined by the BCA method. Then, $150 \mu \mathrm{g}$ of protein in the supernatant was diluted with $50 \mu$ of 'dilution buffer' and mixed with $50 \mu \mathrm{l}$ of $2 \times$ 'reaction buffer' (containing $10 \mathrm{mM}$ DTT) together with $5 \mu \mathrm{l}$ of $4 \mathrm{mM}$ Asp-Glu-Val-Asp-p-nitroanilide (DEVD-pNA). After incubation for $2 \mathrm{~h}$ at $37^{\circ} \mathrm{C}$, the amount of released pNA was measured by absorbance at $405 \mathrm{~nm}$ on an ELISA reader.

\section{Reverse transcription (RT)-PCR analysis of total RNA}

Total RNA of rat chondrocytes was isolated using TRI reagent (Molecular Research Center, Cincinnati, $\mathrm{OH}$ ) following the protocol recommended by the manufacturer. Three hundred $\mathrm{ng}$ of RNA was reverse transcribed and the resulting CDNA was amplified using a one-step RT-PCR kit (Qiagen, Stanford Valencia, CA). Reverse transcription was performed using a thermal program of $50^{\circ} \mathrm{C}$ for $1 \mathrm{~h}$, coupled with $95^{\circ} \mathrm{C}$ for $15 \mathrm{~min}$. PCR reactions were carried out using a thermal cycle program of $94^{\circ} \mathrm{C}$ for $30 \mathrm{~s}, 52^{\circ} \mathrm{C}$ for $60 \mathrm{~s}$, and $72^{\circ} \mathrm{C}$ for $60 \mathrm{~s}$ for 30 repeating cycles. GAPDH was used as a reference for the semiquantitative analysis of gene expression. The nucleotide sequences of the primers were: Fas forward primer: 5'-GAATGCAAGGGACTGATAGC-3', Fas reverse primer: 5'-TGGTTCGTGTGCAAGGCTC-3', FasL forward primer: 5'-GGAATGGGAAGACACATATGGAACTGC-3', FasL reverse primer: 5'CATATCTGGCCAGTAGTGCAGTAATTC-3', collagen type II forward primer: 5'-AGGAGGCTGGCAGCTG-3', collagen type II reverse primer: 5'CACTGGCAGTGGCGAG-3', GAPDH forward primer: 5'-CAAGCTCCTACCATTCATGC-3', GAPDH reverse primer: 5'-TTCACACCGACCTTCACC-3'. PCR products were resolved by agarose gel $(2 \%)$ electrophoresis and visualized with ethidium bromide under UV light.

\section{Cell viability test}

The viability of chondrocytes was determined using an MTT assay to assess non-viable cells, i.e. apoptosis (Mansfield et al., 1999). The assay is based on the enzymatic activity of mitochondrial dehydrogenases reacting with pale yellow tetrazolium salt 3-(4,5-dimethylthiazol-2-yl)-2,5-diphenyItetrazolium bromide (MTT), producing a dark blue formazan product. To perform the analysis, MTT $(120 \mu \mathrm{g} / \mathrm{ml})$ was added to the serum-free medium and the cells were cultured for $3 \mathrm{~h}$ at $37^{\circ} \mathrm{C}$. After removing the culture medium, the product was solubilized in dimethyl sulfoxide (DMSO) and the absorbance at $540 \mathrm{~nm}$ was read on an ELISA reader.

\section{Flow cytometry}

To investigate the effect of guanosine on the 


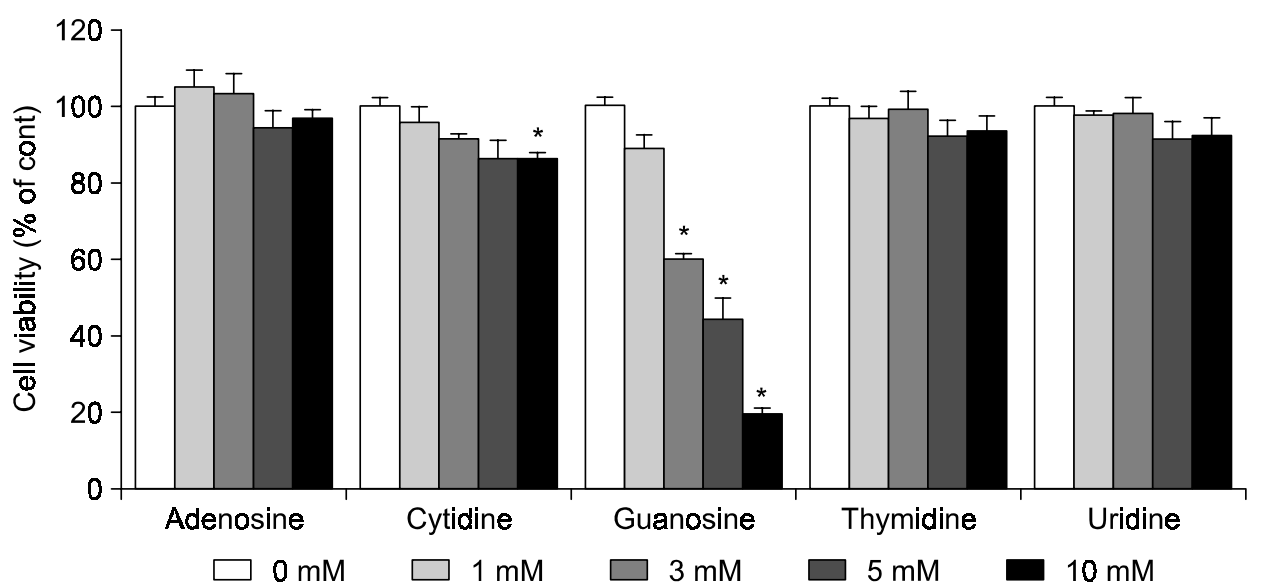

A

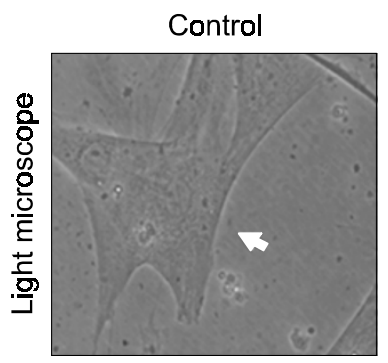

Guanosine (3 mM)

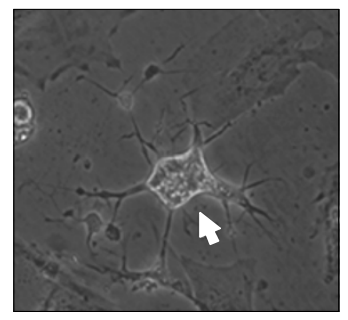

B

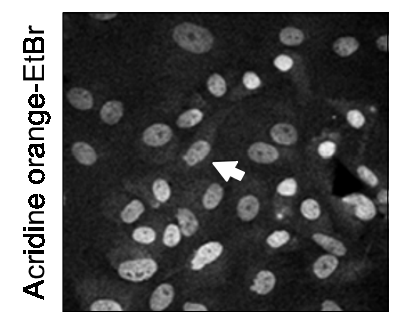

Figure 1. The effects of guanosine on the growth of chondrocytes in culture evaluated by cell viability. Rat chondrocytes (1 $\times 10^{4}$ cells per well) were cultured for $48 \mathrm{~h}$ in a 96-well culture plate in the absence or presence of guanosine. The dose-dependent effects of guanosine were assessed by an MTT assay. The mean values and SDs from three independent analyses are shown. ${ }^{*} P<0.01$.

C

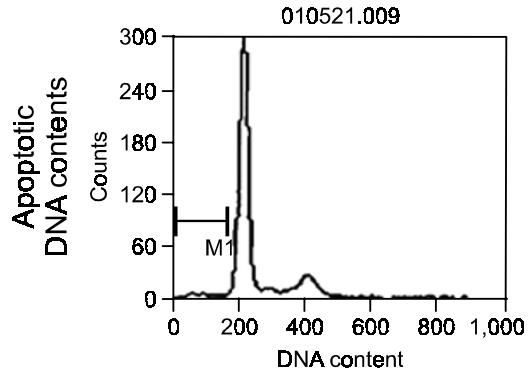

Sub-G1 fraction :

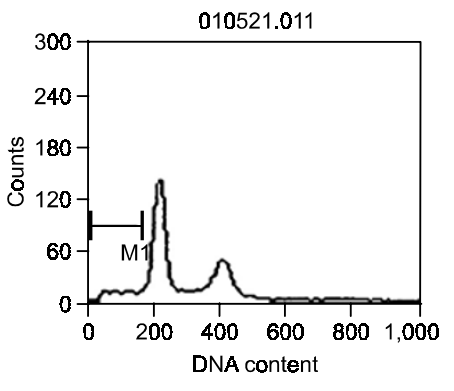

$8.46 \%$

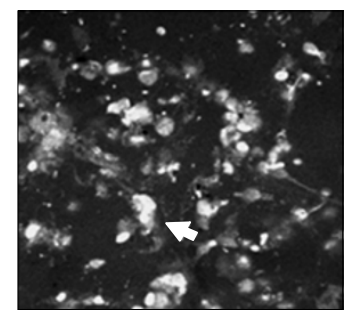

Figure 2. Effects of guanosine on chondrocyte apoptosis. (A) Light microscopic observation of chondrocytes cultured for $48 \mathrm{~h}$ in the absence (control) and presence of guanosine ( $3 \mathrm{mM})$. (B) Acridine orange-ethidium bromide treated chondrocytes. (C) DNA content in the sub-G1 fraction in flow cytometry.

apoptosis of chondrocytes, the DNA content of the cells at a given stage of the cell-cycle was determined by flow cytometry, according to the method previously described by Noguchi et al., (1981). Chondrocytes were cultured with a desired concentration of guanosine for $48 \mathrm{~h}$ and fixed with $70 \%$ ethanol. The cells were then treated with 0.25 $\mu \mathrm{g} / \mathrm{ml}$ of RNase and stained with $50 \mu \mathrm{g} / \mathrm{ml}$ of propidium iodide. The DNA content of the chondrocytes was then measured using a FACScaliber system (Becton Dickinson, Franklin Lakes, NJ).

\section{Statistical analysis}

Statistical analyses were performed using Student's paired $t$-test $(P$ values are reported in the figure legends). At least 3 independent analyses were performed for each test.

\section{Results}

\section{Induction of chondrocyte apoptosis by guanosine}

We investigated the effect of guanosine on the growth of chondrocytes by means of an MTT assay. As shown in Figure 1, an increase in the concentration of guanosine resulted in a dose-dependent decrease in the number of viable chondrocytes, indicating significant cell death within $48 \mathrm{~h}$. On the other hand, adenosine, cytidine, thymidine and uridine at various concentrations did not appear to affect the growth of chondrocytes. 


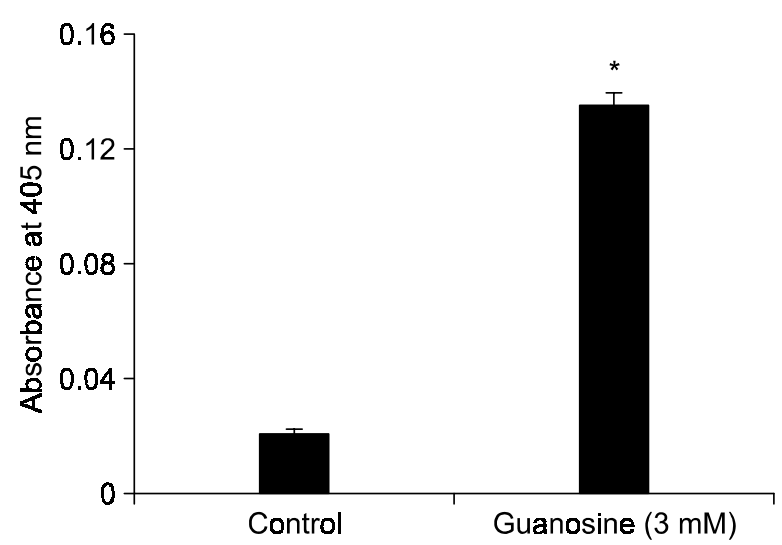

Figure 3. Effects of guanosine on the apoptotic process of chondrocytes evaluated by a caspase-3 activity assay. Chondrocytes were cultured for $36 \mathrm{~h}$ in the absence or presence of guanosine. The cell lysates were incubated with Ac-DEVD-pNA for $4 \mathrm{~h}$ at $37^{\circ} \mathrm{C}$. ${ }^{*} P<0.05$.
Condrocytes cultured in the absence of guanosine maintained their original nuclear morphology throughout the culture period (Figure 2A and B). Staining of dead cells with acridine orange-ethidium bromide revealed condensation and fragmentation of the nuclei, which are typical manifestations of apoptosis (Figure 2B). Subsequent FACS analysis of the cells showed an increasingly larger sub-G1 fraction with presence of $3 \mathrm{mM}$ guanosine in the culture (Figure 2C).

Since caspase- 3 is an executioner of apoptosis by a variety of stimuli (Earnshaw et al., 1999), we investigated the possibility that caspase-3 is involved in guanosine-induced apoptosis of chondoryctes. Culturing chondrocytes for $36 \mathrm{~h}$ in $3 \mathrm{mM}$ guanosine increased the caspase- 3 activity in the chondrocytes 7 -fold compared to a control system (Figure 3).

\section{A}

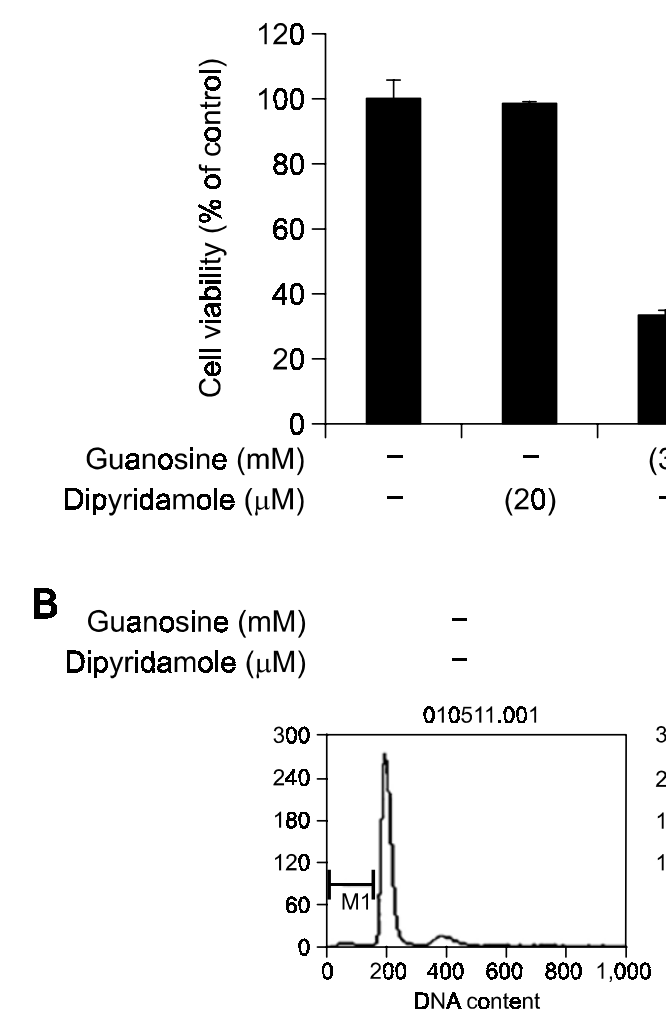

Sub-G1 fraction :

$2.11 \%$
(3)

$-$
(3)

(20)

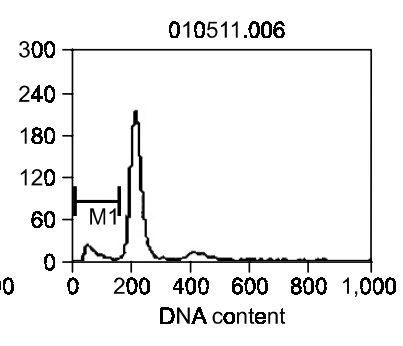

$8.48 \%$
(3)

(20)

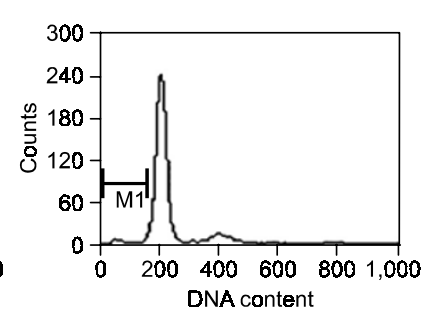

$2.73 \%$

Figure 4. Effects of a transporter blocker on guanosine-induced chondrocyte apoptosis. (A) Dipyridamole inhibits guanosine-induced cell death, as determined by a cell viability assay, (B) DNA content analysis of the sub-G1 fraction by flow cytometry. Cells were pretreated with dipyridamole $(20 \mu \mathrm{M})$ for $1 \mathrm{~h}$, followed by coincubation with guanosine for an additional $48 \mathrm{~h}$. ${ }^{*} P<0.01$. 


\section{Intracellular induction of guanosine in chondrocyte apoptosis}

To determine whether the apoptosis of chondrocytes is mediated by guanosine as a transporter or as a receptor, guanosine-treated chondrocytes were incubated with dipyridamole, which is a nucleoside analog capable of specifically inhibiting transporter activity. A cell viability assay and flow cytometry analysis revealed significant suppression of guanosine-induced apoptosis by dipyridamole (Figure $4 A$ and $B)$.

\section{Induction of Fas ligand by guanosine}

To understand the mechanisms by which guanosine induces apoptosis in chondrocytes, we examined the expressions of apoptosis regulating genes by the RT-PCR method. Fas/Fas ligands are regarded as autocrine receptor-ligands associated with cell death in their signal transduction pathway (Belka et al., 1998). Culturing chondrocytes in the presence of guanosine produced transient expression of the Fas ligand within chondrocytes such that maximal expression of the Fas ligand was observed after $4 \mathrm{~h}$.
Fas ligand induction was not observed in the control system over the same time period (Figure 5A). Fas mRNA, however, was expressed constitutively in cultures both with and without guanosine (Figure $5 \mathrm{~A})$. Thus, the secretion and autocrine engagement of the Fas ligand with Fas at the cell surface are probably linked to induction of chondrocyte apoptosis by guanosine. As a test, cells were preincubated prior to addition of guanosine with the fusion molecule Fas-Fc, which is known to interfere with the Fas-Fas ligand interaction and thereby inhibit the signals originating from Fas (Yamaoka et al., 2000). Fas-Fc $(10 \mu \mathrm{g} / \mathrm{ml})$ was able to block guanosineinduced chondrocyte apoptosis (Figure 5B).

\section{Discussion}

Endochondral ossification involves an ordered progression from cell division through hypertrophic differentiation to cell death (Gibson, 1998). However, the mechanism of cell death in cartilage has not been elucidated owing to the characteristic of cartilage as a single cell type that is not innervated, vascularized, or penetrated by lymphatic vessels

A
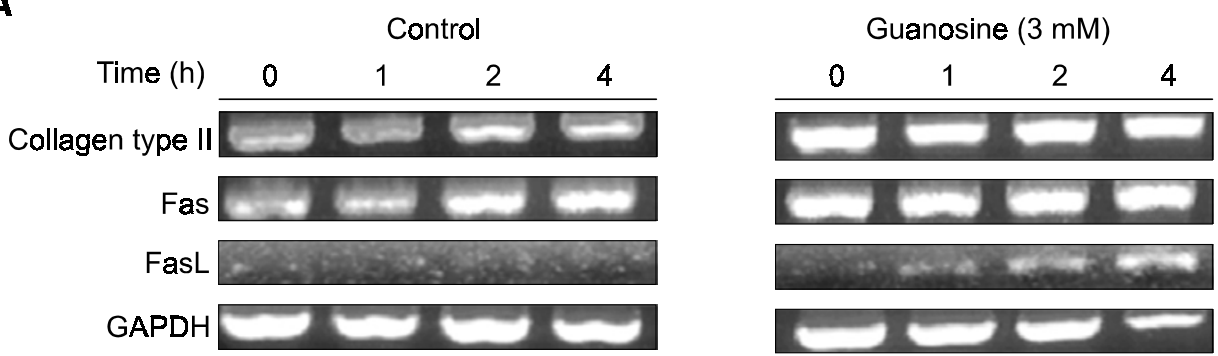

B

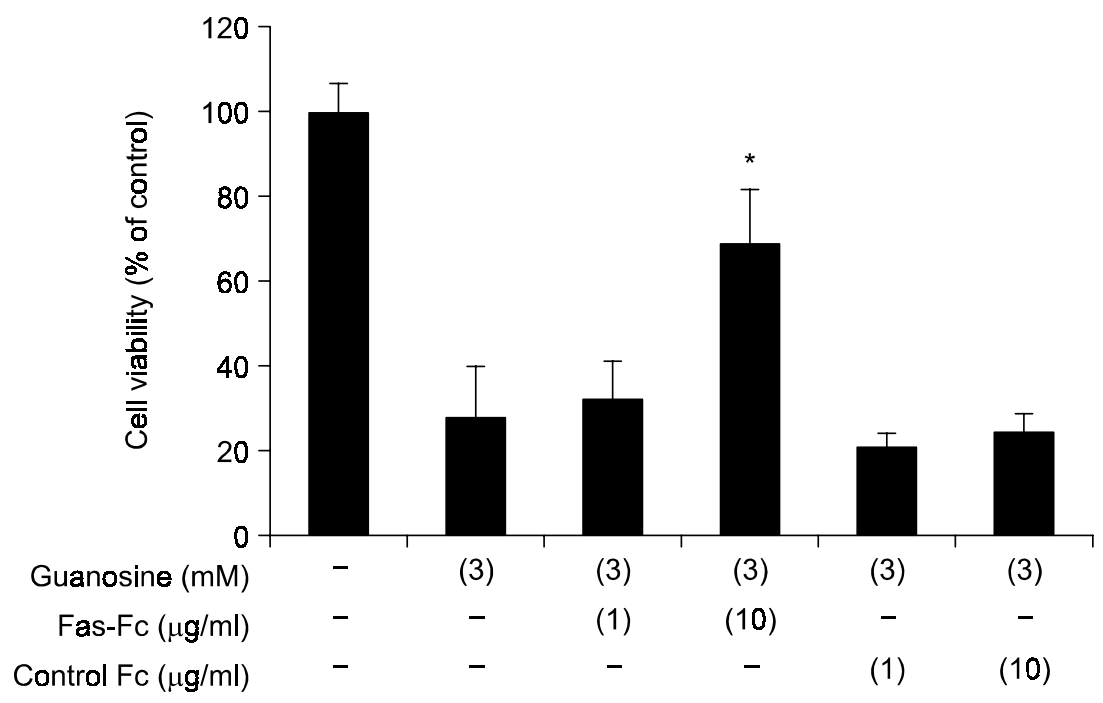

Figure 5. Induction of the Fas ligand in guanosine-induced chondrocyte apoptosis. (A) RT-PCR analysis of FasL mRNA levels in chondrocytes cultured in the absence (control) or presence of guanosine $(3 \mathrm{mM})$ and harvested at various times during the culture. The analysis compared the effects of collagen type II, Fas, FasL, and GAPDH. (B) Inhibition test of FasL-induced apoptosis of chondrocytes by Fas-Fc measured in terms of cell viability. The chondrocytes were incubated in the presence of guanosine $(3 \mathrm{mM})$ for $48 \mathrm{~h}$. Fas-Fc at various concentrations or control Fc at corresponding concentrations were added 30 min prior to addition of guanosine. The results shown are the mean values of three independent analyses. ${ }^{*} P<0.05$. 
(Ishizaki et al., 1994). Matsumoto et al. reported that guanosine concentrations were substantially increased in the hypertrophic zone and in calcified cartilage (1988). We believe that the high levels of guanosine in those areas are linked with chondrocyte physiology, especially apoptosis. Based on the results of an MTT assay (Figure 1), guanosine induced the cell death of chondrocytes in a dose-dependent manner. The effect of guanosine for induction of apoptosis was confirmed by light microscopy, fluorescence microscopy with acridine orange/ethidium bromide dual-staining, FACS analysis, and a caspase- 3 activity assay (Figure 2 and 3 ). Additionally, the effect of guanosine for induction of apoptosis was confirmed by dipyridamole, a guanosine transporter blocker that caused inhibition of guanosine induced chondrocyte apoptosis (Figure 4). Thus, guanosine can induce apoptosis within chondrocyte cells by activating the signal pathway leading to apoptosis.

We investigated whether or not guanosine activated of the extrinsic death pathway rather than the intrinsic pathway as a mechanism for chondrocyte apoptosis. Fas/FasL was used as a candidate extrinsic cell death system because the autocrine Fas ligand was reported to promote apoptosis in peripheral lymphocytes (Belka et al., 1998). Under normal conditions, other cell types do not enter cartilage and chondrocytes do not migrate within the matrix of the cartilage (Hashimoto et al., 1998). The presence of guanosine in the culture increased FasL levels in chondrocytes, concomitant with the induction of apoptosis (Figure 5). Apparently guanosine induces chondrocyte apoptosis by activating the Fas/FasL pathway.

We reported in a previous study that purine nucleoside-induced apoptosis in HL-60 human leukemia cells was modulated by addition of pyrimidine nucleosides (Kim et al., 1998). Although the effect of an unbalanced nucleoside was not fully explainable; some changes were attributed to induction of $\mathrm{Nm} 23$ and/or myc (Kim et al., 1998). The importance of a nucleoside balance at the extreme upstream position of the apoptotic pathway has also been suggested for modulating apoptosis induction (Kang et al., 2001). These reports indicate that a metabolic disturbance of nucleosides may lead to apoptosis. The results of this study provide the first evidence that an intermediate metabolic product participates in the control process of endochondral ossification. The present findings should stimulate research leading to intervention or treatment of age-associated osteoarthritis by manipulating the guanosine content in chondrocytes.

In conclusion, metabolic regulation by the hypertrophic and calcified zone of cartilage generates guanosine, which plays an important role in controlling the apoptotic process in chondrocytes. Condrocyte apoptosis may be mediated by the FasL pathway. The potential for using guanosine as a target for development of novel treatment modalities for osteoarthritis should be investigated.

\section{Acknowledgement}

The authors thank Dr. Yung Dai Kim for his comments on the manuscript. This work was supported by grants from the Korea Research Foundation for Health Science and the Aging and Apoptosis Research Center of the Korea Ministry of Science and Technology (R II 2002-097-05001-0 and R II 2002-097-0001-0).

\section{References}

Belka C, Marini P, Budach W, Schulze-Osthoff K, Lang F, Gulbins E, Bamberg M. Radiation-induced apoptosis in human lymphocytes and lymphoma cells critically relies on the up-regulation of CD95/Fas/APO-1 ligand. Radiat Res 1998; 149:588-95

Earnshaw WC, Martins LM, Kaufmann SH. Mammalian caspases: structure, activation, substrates, and functions during apoptosis. Annu Rev Biochem 1999;68:383-424

Folkman J, Haudenschild CC, Zetter BR. Long-term culture of capillary endothelial cells. Proc Natl Acad Sci USA 1979; 76:5217-21

Gibson G. Active role of chondrocyte apoptosis in endochondral ossification. Microsc Res Tech 1998;43:191-204

Griffith DA, Jarvis SM. Nucleoside and nucleobase transport systems of mammalian cells. Biochim Biophys Acta 1996; 1286:153-81

Hashimoto S, Ochs RL, Rosen F, Quach J, McCabe G, Solan J, Seegmiller JE, Terkeltaub R, Lotz M. Chondrocyte-derived apoptotic bodies and calcification of articular cartilage. Proc Natl Acad Sci USA 1998;95:3094-9

Ishizaki Y, Burne JF, Raff MC. Autocrine signals enable chondrocytes to survive in culture. J Cell Biol 1994;126:1069-77

Izumi T, Shida J, Jingushi S, Hotokebuchi T, Sugioka Y. Administration of growth hormone modulates the gene expression of basic fibroblast growth factor in rat costal cartilage, both in vivo and in vitro. Mol Cell Endocrinol 1995;112:95-9

Kang CM, Suh Y, Jang IS, Park SC. Thymidine-dependent attenuation of the mitochondrial apoptotic pathway in adenosine-induced apoptosis of HL-60 cells. J Cancer Res Clin Oncol 2001;127:570-6

Kim KT, Yeo EJ, Choi H, Park SC. The effect of pyrimidine nucleosides on adenosine-induced apoptosis in HL-60 cells. J Cancer Res Clin Oncol 1998;124:471-7

Mansfield K, Rajpurohit, R, Shapiro IM. Extracellular phosphate ions cause apoptosis of terminally differentiated epiphyseal chondrocytes. J Cell Physiol 1999;179:276-86 
Matsumoto H, DeBolt K, Shapiro IM. Adenine, guanine, and inosine nucleotides of chick growth cartilage: relationship between energy status and the mineralization process. J Bone Miner Res 1988;3:347-52

Noguchi PD, Johnson JB, Browne W. Measurement of DNA synthesis by flow cytometry. Cytometry 1981;1:390-3

Poole AR. The growth plate: Cellular physiology, cartilage assembry, and mineralization. In Cartilage: Molecular Aspects (Hall B , Newman S , eds), 1991, 179-211, CRC Press, Boca Raton, FL

Wright E, Hargrave MR, Christiansen J, Cooper L, Kun J,
Evans T, Gangadharan U, Greenfield A, Koopman P. The Sry-related gene Sox9 is expressed during chondrogenesis in mouse embryos. Nat Genet 1995;9:15-20

Yamaoka M, Yamaguchi S, Suzuki T, Okuyama M, Nitobe J, Nakamura N, Mitsui Y, Tomoike H. Apoptosis in rat cardiac myocytes induced by Fas ligand: priming for Fas-mediated apoptosis with doxorubicin. J Mol Cell Cardiol 2000;32: 881-9

Zuscik MJ, D'Souza M, lonescu AM, Gunter KK, Gunter TE, O'Keefe RJ, Schwarz EM, Puzas JE, Rosier RN. Growth plate chondrocyte maturation is regulated by basal intracellular calcium. Exp Cell Res 2002;276: 310-9 